\title{
Dimensional reduction of electromagnetic boundary value problems
}

Pasi Raumonen ${ }^{1 *}$, Saku Suuriniemi ${ }^{2}$ and Lauri Kettunen ${ }^{2}$

* Correspondence: pasi. raumonen@tut.fi

'Department of Mathematics,

Tampere University of Technology, P.O. Box 553, Tampere Fl-33101,

Finland

Full list of author information is available at the end of the article

\begin{abstract}
This paper develops a systematic and formal approach to dimensional reduction of electromagnetic boundary value problems. The approach is based on the concept of continuous symmetry, and the definitions and the mathematical structures used are conceptually distinct and completely coordinate-free and independent of dimensions. The approach leads to sufficient conditions for when a boundary value problem can be solved as a lower-dimensional one and it shows how to systematically formulate the lower-dimensional problems. The symmetries are described with Lie groups that are products of connected 1-D Lie groups.
\end{abstract}

\section{Introduction}

Symmetric boundary value problems (BVPs) are commonplace in electromagnetics and generally in science and engineering. They include problems with discrete symmetries such as rotational symmetries in rotating electrical machines and problems with continuous symmetries such as continuous translations in waveguides. Continuous symmetries are the key to dimensional reduction of BVPs, which includes 2-D modeling. Even though discrete symmetries are well understood [1], often dimensional reduction is applied ad hoc without a systematic approach based on the underlying principles. In this paper, we explain basic concepts of symmetry and dimensional reduction and give the necessary definitions. We specifically focus on linear electromagnetic BVPs, but the results hold for any problems where the fields are modeled as differential forms and the differential equations are expressed in terms of the exterior derivative. Moreover, we state sufficient conditions for dimensional reduction in such BVPs and present the systematic formulation of the lower-dimensional problems.

In intuitive terms, symmetry is about something remaining the same under some transformations. Thus, symmetry can be used to describe invariances and redundancies, which in turn can be exploited to reduce the complexity of the problem. Observe that "something remaining the same under some transformations" need not depend on coordinates, metric, dimension or orientation of the space. Thus, the usual view of symmetry in engineering under distance-preserving transformations, such as translations or rotations, is a very restricted view of symmetry. Furthermore, dimensional reduction is usually based on the assumption that some components of fields are zero in some special coordinate system. In contrast, our exposition of symmetry and dimensional reduction does not presume distance-preserving transformations or zero field

\section{SpringerOpen ${ }^{\circ}$}

(C) 2011 Raumonen et al; licensee Springer. This is an Open Access article distributed under the terms of the Creative Commons Attribution License (http://creativecommons.org/licenses/by/2.0), which permits unrestricted use, distribution, and reproduction in any medium, provided the original work is properly cited. 
components in some special coordinate system. Although the symmetries of BVPs are defined similarly for discrete and continuous symmetries, systematic application of continuous symmetries (dimensional reduction) requires additional structure called observer. The observers decompose the fields, and all the operators on fields such that the original symmetric BVP can be stated on a lower-dimensional domain.

We employ the mathematical tools of differential geometry because they offer conceptually clear coordinate-free tools that are suitable for all dimensions. A systematic approach to symmetry of BVPs with formal but clear definitions of the essential concepts can help to recognize and apply symmetries that are not intuitively clear at the first sight.

\section{Symmetry}

Let us next express symmetry in formal terms. "Something remaining the same" is expressed with an equivalence relation $\sim$ on set $X$. "Under some transformations" is expressed with a group $(G, \cdot)$ and its left group action on $X$, which is a mapping $F: G$ $\times X \rightarrow X$ such that $F(g \cdot h, x)=F(g, F(h, x))$ and $F(e, x)=x$ hold for all $x \in X$, for all $g, h \in G$ and for the identity $e$ of $G$. Now, the symmetry is defined as follows:

Definition 1. (Symmetry predicate) Let $X$ be a set and $\sim$ an equivalence relation on $X$. Furthermore, let a group $G$ act on $X$ by the action $F: G \times X \rightarrow X$. The relation $\sim$ is $(G, F)$-symmetric over $X$ if $F(g, x) \sim x$ holds for all $x \in X$ and for all $g \in G$.

We also say that the set $X$ is $(G, F, \sim)$-symmetric if the symmetry predicate holds. The axioms of action $F$ imply that the mappings $F_{g}: X \rightarrow X$, defined by $F_{g}(x)=F(g$, $x$ ), are bijections that form a group under the composition of mappings. The mappings $F_{g}$ and the group they form are called the symmetry transformations and the symmetry group of the relation $\sim$. Furthermore, the group actions we consider are effective: For each $g \in G$, there exists a unique $F_{g}$, i.e., $F_{g}=F_{h}$ holds only if $g=h$ holds. Then, we can identify $F_{g}$ with $g$ or denote $F_{g}$ simply by $g$. Moreover, then the symmetry group (group of symmetry transformations $F_{g}$ ) is isomorphic to $G$, and thus, we also denote it by $G$.

Next, let us look at an example of symmetry. If the figure in left of Figure 1 is transformed by rotation of $180^{\circ}$ around its center in clockwise or counter-clockwise, or by reflection w.r.t. its diagonals, then its points are mapped to points of same color. Thus, in terms of the symmetry predicate, the set $X$ is the figure, symmetry transformations $F_{g}$ are the rotations and reflections of the figure, and $\sim$ means the same color of the points.

The orbit of a point $x \in X$ is the set $G x=\{g(x) \in X \mid g \in G\}$, where the symmetry transformations of $G$ map the point $x$. Clearly, by the symmetry predicate, the points
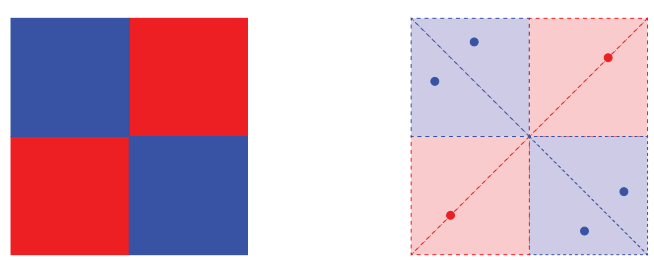

Figure 1 Left: An example of visual symmetry. Right: The blue points are a four-point orbit, and the red points are a two-point orbit. 
of $G x$ are equivalent under . Moreover, each point belongs to exactly one orbit, and thus, orbits define another equivalence relation on $X$.

For example, in the case of Figure 1, when $G$ contains the $180^{\circ}$ rotations and reflection, the orbits are sets of four points, except the points at diagonals, whose orbits contain two points (see right of Figure 1). The center point is the only point in its orbit.

Symmetry of a BVP implies that the unknown fields can be solved from a reduced BVP defined on a reduced domain: Its solution can be expanded to a solution of the original BVP using the symmetry transformations and the equivalence relation. Similarly, the square on Figure 1 can be reconstructed from only a part of the original by the rotations and reflections and the equivalence relation. Thus, the "bigger" the symmetry group is, the more the problem can be reduced and savings gained on the computational resources.

\section{Differential geometry}

A fluent explanation for symmetry and particularly the dimensional reduction of electromagnetic BVPs requires clear separation of different aspects such as metric and dimension. The structures of differential geometry meet the requirements. This section gives a brief introduction to some of the essential structures we use in this paper. References with precise definitions and more detailed expositions include [2-6].

Differentiable manifolds serve as the domains of BVPs. For each $m$-manifold $M$, there exists a class of homeomorphisms $U \subset M \rightarrow \mathbb{R}^{m}$ called charts. Each point $x$ of $M$ has a tangent space $T_{x}(M)$, which is an $m$-dimensional vector space of tangent vectors. Any differentiable mapping $f: M \rightarrow N$ between manifolds induces a unique mapping $f_{*,}$, the pushforward of $f$, that maps linearly from $T_{x}(M)$ to $T_{f(x)}(N)$. An oriented manifold is a manifold whose tangent spaces are oriented and a manifold-with-boundary $M$ is a manifold that has a boundary $\partial M \subset M$. A diffeomorphism is a differentiable bijection between manifolds with a differentiable inverse. An embedded submanifold of $M$ is a pair $(N, f)$, where $N$ is a manifold and $f: N \rightarrow f(N) \subset M$ is a diffeomorphism.

Dimensional reduction is based on "smooth symmetries." This smoothness is reflected in the manifold structure of the symmetry groups. A Lie group is a group that is also a manifold such that the group operations are differentiable mappings. An example of a Lie group is $\mathbb{R}=(\mathbb{R},+, i d)$, where $\mathbb{R}$ is the set of real numbers, the group operation + is the addition of real numbers, and the chart $i d$ is the identity mapping of $\mathbb{R}$. Another example is $S^{1}$, the group of all complex numbers with modulus one under multiplication. $\mathbb{R}$ and $S^{1}$ are the only connected 1-D Lie groups up to isomorphism, and every connected 2-D Lie group is a product of them.

It is possible to define an analysis on manifolds in a coordinate- and metric-free manner and independent of the dimension. This analysis employs differential forms: A differential $p$-form $\omega$, or a $p$-form for short, assigns each point $x \in M$ an antisymmetric $p$-linear mapping $\omega_{x}$ from the tangent space $T_{x}(M)$ to the real numbers. The vector space of all $p$-forms on $M$ is denoted by $\Omega^{p}(M)$, and the set of all differential forms on $M$ is denoted by $\Omega(M)$. For each differentiable mapping $f: M \rightarrow N$ between manifolds, there is an induced mapping $f^{*}: \Omega(N) \rightarrow \Omega(M)$ called the pullback. It is defined point-wise as follows: $\left(f^{*} \omega\right) x\left(v_{1}, \ldots, v_{p}\right)=\omega_{f(x)}\left(f_{*} v_{1}, \ldots, f_{*} v_{p}\right)$ holds for all $x \in M$, 
$v_{1}, \ldots, v_{p} \in T_{x}(M)$. The restriction of differential forms to a submanifold $A$ of $M$ is given by the trace $t_{A}$, which is the pullback $i_{A}^{*}$ of the inclusion map $i_{A}: A \rightarrow M$.

The contraction $i_{X}$ by a vector field $X$ decreases the degree of a form $\omega$ by one such that $\left(i_{X} \omega\right)_{x}\left(v_{2}, \ldots, v_{p}\right)=\omega_{x}\left(X_{x}, v_{2}, \ldots, v_{p}\right)$ holds for all $x \in M, v_{2}, \ldots, v_{p} \in T_{x}(M)$. The wedge product is a bilinear mapping $\Lambda: \Omega(M) \times \Omega(M) \rightarrow \Omega(M)$ which is anticommutative. The extension $I_{\alpha}$ by a 1 -form $\alpha$ and the wedge product increases the degree of $\omega$ by one: $I_{\alpha} \omega=\alpha \Lambda \omega$.

The differential operators grad, curl, and div of the vector analysis are metric counterparts of a single metric-free differential operator on differential forms called the exterior derivative. On a manifold $M$, it is the linear mapping $d_{M}: \Omega^{p}(M) \rightarrow \Omega^{p+1}(M)$ such that it is the differential for 0 -forms and $d_{M}\left(d_{M} \omega\right)=0$ holds for all $\omega$. Furthermore, $d$ commutes with pullback: $f^{*} \circ d_{N}=d_{M} \circ f^{*}$ holds for $f: M \rightarrow N$.

When $(\mathbb{R},+)$ acts on manifold $M$ such that the symmetry transformations are diffeomorphisms, then the symmetry group is called a 1-parameter group of transformations. The action induces a smooth vector field $X$ on $M$ such that the vector field is everywhere tangent to the orbits, most of which are now 1-D submanifolds. With a 1-parameter group of transformations and the pullback, one can define a directional derivative of forms in the direction of the orbits. This derivative is called the Lie derivative, and it is denoted by $\mathcal{L}_{X}$.

To assure uniqueness of a BVP solution, some cohomology classes of the fields may have to be specified explicitly, and by de Rham's theorem, this can be done by fixing the values of integrals of the fields over suitable submanifolds [7]: These integrals are presented as a linear operator $\mathcal{H}$ that operates on fields. Thus, the cohomology condition of a form $\omega$ is given by a real number tuple $\mathcal{H}(\omega)$ that contains the values of the integrals.

A metric tensor allows a definition of Hodge-operator which can be used to express constitutive equations [3]. The Hodge-operator is a linear isomorphism $\star: \Omega^{p}(M) \rightarrow$ $\Omega^{m-p}(M)$ such that it is definite. To give preference to the physics modeled by the constitutive equations over the metric chosen for distance measurements and modeling, we make the following generalization [6]:

Definition 2. Let $M$ be an oriented m-manifold. A definite linear isomorphism $v: \Omega^{p}$ $(M) \rightarrow \Omega^{m-p}(M)$ is a Hodge-like operator if there exists a metric tensor $\varphi$ of $M$ and a linear isomorphism $v_{\varphi}: \Omega^{m-p}(M) \rightarrow \Omega^{m-p}(M)$ such that $v=v_{\varphi}{ }^{\circ \star_{\varphi}}$ holds, where $\star_{\varphi}$ is the Hodge-operator induced by $\varphi$.

\section{Description of model BVP}

In this paper, we want to cover linear electromagnetic BVPs, and thus, we consider BVPs expressible with the exterior derivative and form-independent Hodge-like operators. For simplicity, we consider mainly the following model BVP:

Definition 3. (Model BVP) The domain of model BVP is an oriented m-dimensional manifold-with-boundary M. A pair of fields, a p-form $C$ and an $(m-p)$-form $K$, are governed by the differential equations expressed with the exterior derivative $d_{M}$ and connected to each other by a Hodge-like operator $v$. The source field is given by a $(p+$ 1)-form $Q$. The boundary values $c$ and $k$ of $C$ and $K$, respectively, are given as restrictions $t_{\partial M^{1}}$ Cand $t_{\partial M^{2}}$ Kto the complementary parts $\partial M^{1}$ and $\partial M^{2}$ of the boundary $\partial M$. 
The cohomology conditions $\mathcal{H}(C)$ and $\mathcal{H}(K)$ of $C$ and $K$ are given by real number tuples $k_{\mathcal{H}}$ and $k_{\mathcal{H}}$.

Thus, model BVP consists of the following equations:

$$
\begin{array}{llll}
d_{M} C=Q, & K=v C, & t_{\partial M^{1}} C=c, & \mathcal{H}(C)=c_{\mathcal{H}}, \\
d_{M} K=0, & & t_{\partial M^{2}} K=k, & \mathcal{H}(K)=k_{\mathcal{H}},
\end{array}
$$

which hold in $M . \square$

Model BVP encompasses, e.g., magnetostatic problems: $M$ is a 3-manifold-withboundary, $C$ is the magnetic field 1 -form $H, K$ is the magnetic flux density 2 -form $B$, $Q$ is the current density 2 -form $J, v$ models permeability $\mu$, and $c_{\mathcal{H}}$ and $k_{\mathcal{H}}$ are tuples of real numbers that describe the magnetomotive forces of $H$ and fluxes of $B$ over some curves and surfaces. Moreover, Maxwell's equations over spacetime manifold are included in the model BVP [8].

Remark 1. The reader may notice that quasi-static BVPs or the complete set of four Maxwell's equations with time as independent parameter are not included in the model $B V P$. However, the theory we represent for the model BVP can be extended to more general BVPs, including quasi-static cases and full Maxwell's equations. Particularly, BVPs can contain many pairs of fields that are connected to each other by a Hodge-like operator. The differential equations for the fields may contain also time derivative operator $\partial_{t}$ in addition to the exterior derivative, and there may be additions of fields. For example, in electromagnetism, we have the equation $d_{M} H=J+\partial_{t} D$, where $D$ is the electric flux density 2-form and either J or $\partial_{t} D$ can be given as the source field.

\section{Symmetric BVPs}

In this section, we consider BVPs included in the model BVP and define the invariance (symmetry) of the fields, differential equations, boundary values, Hodge-like operators, and cohomology conditions. Finally, we state a theorem stating that the unique solution of a symmetric BVP is symmetric.

\subsection{The symmetry transformations of the domain}

We consider effective group actions whose symmetry transformations are diffeomorphisms of the BVP domain $M$. For dimensional reduction, we assume that the symmetry group $G$ is also a Lie group that is a product of connected 1-D Lie groups. Thus, the symmetry group $G$ is isomorphic to a product Lie group whose product factors are all either $\mathbb{R}$ or $S^{1}$. For example, in cylindrical symmetry, $G$ is isomorphic to $\mathbb{R} \times S^{1}$, and most orbits are cylindrical surfaces. The axis of rotation forms an orbit whose dimension is smaller than that of the other orbits, see Figure 2.

This is because the action is effective but not free: There are symmetry transformations other than the identity mapping that map some points to themselves. These special orbits are called singular orbits.

Definition 4. Let group $G$ act effectively on manifold $M$. A point of $M$ is singular if it is a fixed point for a symmetry transformation that is not the identity mapping of $M$. An orbit is singular if it contains a singular point.

\subsection{Invariance of fields}

With a group action on manifold $M$, we can geometrically characterize invariant fields: The symmetry transformations $g$ of $M$ define correspondences between the points of 


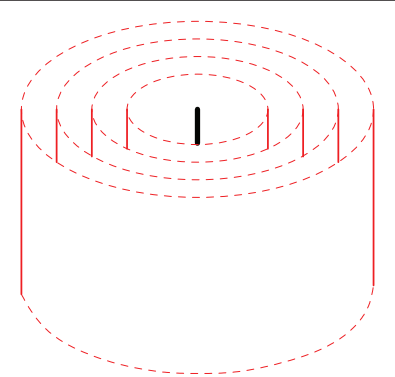

Figure 2 Singular orbit. Sections of infinitely long cylindrical surfaces that are orbits under a cylindrical action (rotations and translations). The axis of rotation (black line) forms a singular orbit because all the rotations map the points of the axis to themselves.

$M$, and the pushforwards of $g$ define correspondences between the tangent vectors at corresponding points. Then, a differential form is invariant if at corresponding points, it returns the same number for corresponding tangent vectors. Thus, a $G$-invariant form $\omega$ equals to its pullback under the symmetry transformations, i.e., $g^{*} \omega=\omega$ holds for all $g \in G$. On the other hand, the group action on manifold $M$ induces a group action on the set of differential forms $\Omega(M)$ such that the pullbacks $g^{*}$ are the symmetry transformations of $\Omega(M)$. Furthermore, if the equivalence relation in $\Omega(M)$ is the equality of forms (defined pointwise), then it is G-symmetric over the set of all Ginvariant forms. That is, in terms of the symmetry predicate, the equality of forms is $G$-symmetric in the set of all $G$-invariant forms under these pullbacks.

We consider also more complicated actions on $\Omega(M)$, where the pullback $g^{*}$ is multiplied by a group homomorphism $h: G \rightarrow \mathbb{F}$, where $\mathbb{E}$ is the field of real or complex numbers. For example, in the case of time-harmonic fields, the symmetry transformations $g: M \rightarrow M$ of $G=(\mathbb{R},+)$ are translations in time and $h: G \rightarrow \mathbb{C}$ is a group homomorphism such that $h(g)=e^{i \alpha g}$ holds, where $\alpha$ is some real number. Then, a form $\omega$ is time harmonic with frequency $\alpha$ if $g^{*} \omega=h(g) \omega$ holds for all $g \in G$.

Definition 5. Let a group $G$ act on a manifold $M$ and let $h: G \rightarrow \mathbb{F}$ be a group homomorphism (Lie group homomorphism for Lie group $G$ ). A differential form $\omega$ on $M$ is ( $G$, h)-invariant if $g^{* *} \omega=h(g) \omega$ holds for all $g \in G$. A vector field $X$ on $M$ is $(G, h)$-invariant if $g_{*} X=h(g) X$ holds for all $g \in G$.

If $h(g)=1$ holds for all $g \in G$, then we talk about G-invariance instead of $(G, h)$ invariance.

For dimensional reduction, we need to express the $(G, h)$-invariance of the forms equivalently in local terms. This can be done with the Lie derivative, if the symmetry group $G$ is a connected 1-D Lie group, as assumed: If $G$ is parameterized by real numbers with a Lie group homomorphism $\beta: \mathbb{R} \rightarrow G$, the group action $G \times M \rightarrow M$ is represented as a 1-parameter group of transformations $\mathbb{R} \times M \rightarrow M$ that induces a smooth nonzero $G$-invariant vector field $X_{\beta}$ everywhere tangent to the orbits [[6], p. 101]. With $\beta$, the mapping $h: G \rightarrow \mathbb{F}$ can be represented as a mapping $h_{\beta}: \mathbb{R} \rightarrow \mathbb{F}$ such that $h_{\beta}=h \circ \beta$ holds. Because the vector field $X_{\beta}$ is everywhere tangent to the orbits, the Lie derivative w.r.t. $X_{\beta}$ gives us an equivalent way to state the $(G, h)$-invariance of the fields [[6], p. 102]:

Theorem 1. Let $G$ be a connected 1-D Lie group and $h: G \rightarrow \mathbb{F}$ a Lie group homomorphism. Furthermore, let $\beta: \mathbb{R} \rightarrow G$ be a Lie group homomorphism that 
parameterizes $G$ with real numbers and let $X_{\beta}$ be the induced vector field. For a $(G, h)$ invariant form $\omega$ on manifold $M, \mathcal{L}_{X_{\beta}} \omega=h_{\beta}^{\prime}(0) \omega h o l d s$ everywhere.

To make the notation simpler, we regard $\beta$ implicit and denote $X_{\beta}$ and $h_{\beta}^{\prime}(0)$ simply by $X$ and $h^{\prime}(0)$.

\subsection{Invariance-preserving operators}

The model BVP has the operators $d_{M}, t_{\partial M}, v$, and $\mathcal{H}$. In a symmetric BVP these operators map symmetric fields to symmetric fields, i.e., they should preserve the invariance of fields in the sense that they map $(G, h)$-invariant forms to $(G, h)$-invariant forms. This requires that they commute with the symmetry transformations and preserve the equivalence relation. Because the equivalence relation in $\Omega(M)$ is the equality of the forms, they automatically preserve the equivalence.

Because the exterior derivative $d_{M}$ is linear and commutes with the pullback, it preserves $(G, h)$-invariance under every group $G$ whose symmetry transformations are diffeomorphisms. Although $d_{M}$ is in this sense invariant under diffeomorphism group, differential equations expressed with $d_{M}$ are not generally invariant under the diffeomorphism group: The invariance of a differential equation, such as $d_{M} \omega=\rho$, means that if $\omega$ is a solution, then the transformed solution $g^{* \omega} \omega$ is also a solution or $d_{M} g^{* *} \omega$ $=\rho$ holds [9]. Now, the invariance of the equation $d_{M} \omega=\rho$ is defined by $\rho$ and if $\rho$ is $(G, h)$-invariant, then clearly, the equation is also $(G, h)$-invariant: $h(g) g^{*}\left(d_{M} \omega\right)=h(g)$ $g^{*} \rho$ is equivalent to $d_{M} h(g) g^{* *} \omega=\rho$.

The restriction of a diffeomorphism $F: M \rightarrow M$ to the boundary $\partial M$ is a diffeomorphism $F_{\partial}: \partial M \rightarrow \partial M$ [4]. Thus, a group action on the manifold induces a group action on the boundary, and therefore, the invariance of boundary values is defined similarly as in the above definition of invariant fields. Furthermore, it follows that the boundary values of a $(G, h)$-invariant fields are automatically $(G, h)$-invariant, and thus, the trace $t_{\partial M}$ is invariance-preserving: If $g_{\partial}^{*}$ denotes the restrictions of the pullbacks $g^{*}$ to the boundary, then $t_{\partial M} \circ g^{*}=g_{\partial}^{*} \circ t_{\partial M}$ holds.

Contrary to the operators $d_{M}$ and $t_{\partial M}$, Hodge-like operators do not automatically preserve invariance of fields under any group action, and thus, this must be tested:

Definition 6. A Hodge-like operator $v$ on $M$ is $(G, h)$-invariance-preserving if $g^{*} \circ v=$ $v \circ g^{*}$ and $h(g) v=v h(g)$ hold for all $g \in G$.

Note that the above definition permits so-called anisotropic materials. Furthermore, because a Hodge-like operator is linear, $h(g) v=v h(g)$ holds for all Hodge-like operators.

The cohomology class of a field $\omega$ is restricted by a cohomology condition $\mathcal{H}(\omega)$ that fixes the values of the integrals of $\omega$ over specific submanifolds. The following definition gives the cohomology conditions for $(G, h)$-invariant fields:

Definition 7. The cohomology condition $\mathcal{H}(\omega)$ of a field $\omega$ is (G, h)-invariance-preserving if $\mathcal{H}\left(g^{*} \omega\right)=h(g) \mathcal{H}(\omega)$ holds for all $g \in G$.

If the cohomology condition $\mathcal{H}(\omega)$ fixes the value of the integral of $\omega$ over submanifold $\Gamma$, then $\mathcal{H}(\omega)$ preserves $(G, h)$-invariance of $\omega$ if the integral of $\omega$ over $g(\Gamma)$ equals the integral of $\omega$ over $\Gamma$, multiplied by $h(g)$ :

$$
\int_{g(\Gamma)} \omega=\int_{\Gamma} g^{*} \omega=h(g) \int_{\Gamma} \omega .
$$




\subsection{Unique solution of a symmetric BVP is symmetric}

We state a theorem that guarantees the symmetry of the solution of the model BVP when the source fields, boundary values, Hodge-like operators, and cohomology conditions are symmetric and the BVP has a unique solution ([[6], p. 105] proves this for linear constitutive equations).

Definition 8. A BVP is $(G, h)$-invariant if its source fields and boundary values are $(G, h)$-invariant and the Hodge-like operators and the cohomology conditions are $(G$, h)-invariance-preserving.

Theorem 2. If the model BVP is $(G, h)$-invariant and has a unique solution, then the solution fields $C$ and $K$ are $(G, h)$-invariant.

Remark 2. The above theorem can be extended to more general cases discussed in Remark 1, such as quasi-static problems and problems involving the full set of four Maxwell's equations.

\section{Orbit space}

Due to invariance of fields, it is sufficient to know the fields at one point of each orbit. In order to construct a domain for a reduced BVP, one point from each orbit must be chosen such that the resulting whole is a manifold. These domains, or symmetry cells, are submanifolds of the domain of a symmetric BVP. In dimensional reduction, the symmetry cells are lower-dimensional submanifolds. There is no canonical choice of symmetry cell. However, all the symmetry cells are required to be canonically diffeomorphic. Then, the set of all orbits can be given a canonical manifold structure that is independent of the choice of symmetry cell [[6], p. 109]. With this manifold structure, the set of all orbits is called the orbit space, and it is the canonical domain for the reduced BVPs.

Let us next define the symmetry cells and orbit space formally. The set of all orbits of $M$ under $G$ is denoted by $M / G$. There is a natural projection $\pi: M \rightarrow M / G$ such that each point of $M$ is mapped to its orbit. $\pi$ induces the quotient topology for $M / G$ from $M$ such that the topology is compatible with the orbits: $U \subset M / G$ is open if and only if its preimage $\pi^{-1}(U)$ is an open set of $M$. This topology makes $\pi$ continuous [10], and together with $\pi$, we can now define the symmetry cells (or G-reduced domains as in [6]):

Definition 9. An embedded submanifold-with-boundary $A$ of $M$ is a symmetry cell, if there is a continuous mapping $\kappa: M / G \rightarrow M$, called cross-section, such that $\kappa(M / G)$ $=A$ holds and $\pi \circ \kappa$ is the identity mapping of $M / G$.

$\kappa$ is called cross-section because it maps each orbit to one of its points (see Figure 3).

Each cross-section is a homeomorphism to its range $A$ and induces a manifold structure for $M / G$ by the requirement that the cross-section is a diffeomorphism from $M / G$ to its range. To make the induced manifold structure independent of the choice of the cross-section, all the symmetry cells must be canonically diffeomorphic: A mapping $\kappa_{2} \circ \kappa_{1}^{-1}$ from symmetry cell

$A_{1}$ to symmetry cell $A_{2}$ must be a diffeomorphism. Now, we can define the orbit space.

Definition 10. The set of all orbits $M / G$ together with a differentiable manifold structure is the orbit space, if the manifold structure is induced from a symmetry cell and is independent of the choice of the symmetry cell. 


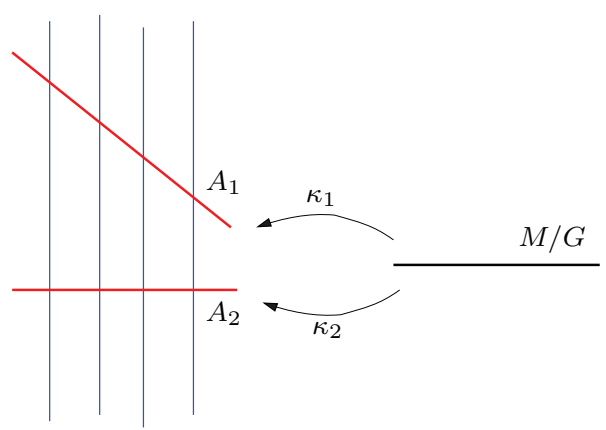

Figure 3 Symmetry cells and orbit space. The blue lines describe orbits, and the red lines describe symmetry cells $A_{1}$ and $A_{2}$ defined by the cross-sections $\kappa_{1}$ and $\kappa_{2}$. The black line describes the orbit space $M / G$.

How are boundaries of $M$ and its orbit space $M / G$ related? Clearly, the orbits of boundary points of $M$ are at the boundary of the orbit space $M / G$. However, the points of orbits that are at the boundary of the orbit space $M / G$ need not be at the boundary of $M$. For example, in the case of rotational symmetry, as in Figure 4, every point of the axis of rotation is on the boundary of some symmetry cell, but not on the boundary of $M$.

The points of the axis belong to singular orbits (Def. 4), and in this paper, we assume that the singular orbits are always at the boundary of the orbit space.

The symmetry of the BVPs is independent of metric, and the reduced BVPs can be defined directly from the original BVP without any metric in the orbit space. This agrees with the fact that there is no canonical metric for the orbit space (see Figure 3, where cross-sections $\kappa_{1}$ and $\kappa_{2}$ induce different metrics).

\section{G-observers and horizontal forms}

This is the point where the development of the theory of dimensional reduction diverges from the theory of discrete symmetries. A dimensional reduction is often carried out with a special coordinate system where one or more of the components of fields are assumed to be zero. However, the symmetry is independent of the coordinates, and it needs not make some components vanish. We build our theory without

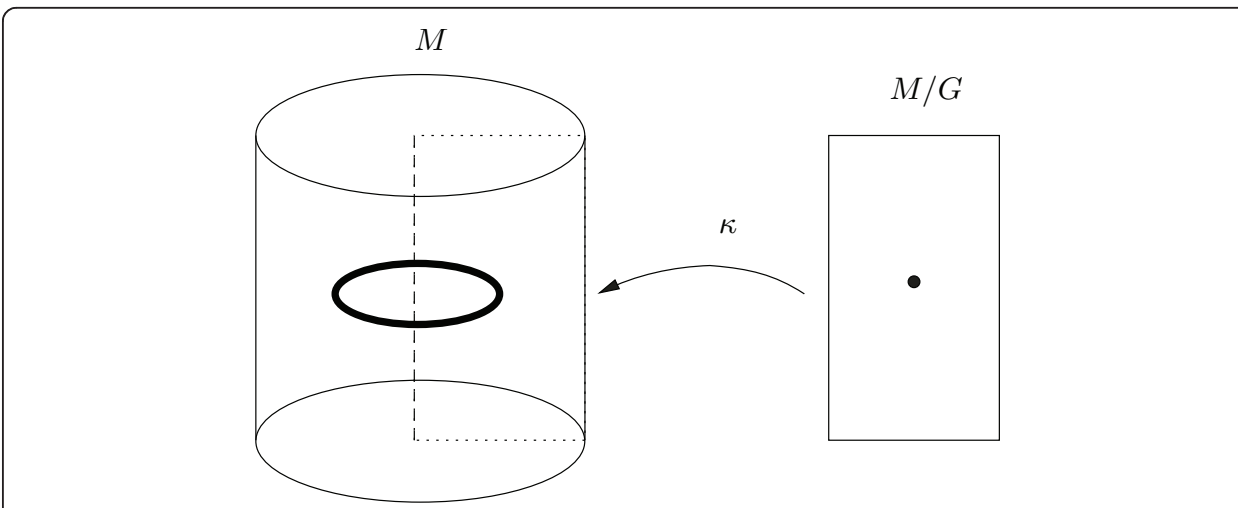

Figure 4 Boundary of an orbit space. $M$ is a 3-D domain that has a rotational symmetry. The thick circle represents a conductor, and the broken line shows the axis of rotation. The points of the axis constitute singular orbits, which are part of the boundary of the 2-D orbit space $M / G$. 
any references to coordinates with a conceptual geometric structure called the G-observer. A G-observer induces two complementary projections that decompose fields into two components. These components are horizontal forms, and $(G, h)$-invariant horizontal forms on $M$ bijectively correspond to differential forms on the orbit space $N=$ $M / G$. Furthermore, the observer-induced projections also decompose the boundary values, the exterior derivative (differential equations), and Hodge-like operators (constitutive equations) such that they are expressed in terms of horizontal operators operating on horizontal forms. These horizontal operators bijectively correspond to operators in $N$ such that decomposed $(G, h)$-invariant BVPs on $M$ bijectively correspond to lower-dimensional BVPs on $N$.

\subsection{G-observers}

The origins of observer structures are in modern physics, where spacetime is decomposed into space and time by an observer $[8,11]$. In the dimensional reduction, 1-D smooth symmetries induce orbits that are 1-D manifolds passing through every symmetry cell. An observer decomposes the fields into two components: the component along the orbits and the component along some symmetry cell. Furthermore, there is a multitude of different possibilities to decompose fields corresponding to different choices of symmetry cells.

Definition 11. Let a 1-D connected Lie group $G$ act on a manifold M. A pair $(T, \tau)$ of a vector field and a 1-form on $M$, respectively, is a G-observer on $M$, if

(1) $T$ and $\tau$ are smooth and G-invariant,

(2) there exists a representation of the action as a 1-parameter group of transformations such that $T$ is the induced vector field ( $T$ is everywhere tangent to the orbits),

(3) there exists a symmetry cell $A$ such that $\tau(v)=0$ holds for all vectors $v$ tangent to A,

(4) $\tau(T)=1$ holds everywhere except at the singular points, where $\tau$ is not defined and $T$ is the zero vector.

Remark 3. For each $g \in G$, the image $g A=\{g(p) \in M \mid p \in A\}$ is a symmetry cell similar to $A$ in the sense that $\tau(v)=0$ holds for all vectors $v$ tangent to gA. Furthermore, because $T$ is smooth and tangent to the orbits, it must be the zero vector at the singular points. At the same time, $\tau$ is not defined at singular points because then $\tau(T)=1$ cannot hold. If $\kappa$ is the cross-section $N \rightarrow A$ defining $A$, then by the above Definition, $\kappa^{*} \tau=$ 0 holds. Finally, it follows from the above Definition that the 1-form $\tau$ of a G-observer is closed or $d_{M} \tau=0$ holds. [[6], p. 115]

\subsection{Decomposition of fields}

A G-observer $(T, \tau)$ define s two complementary projections for fields by contraction and extension: $P_{\tau}=i_{T} \circ I_{\tau}$ and $P_{T}=I_{\tau} \circ i_{T}[6,11,12]$. If $i d$ denotes the identity mapping of $\Omega(M)$, the complementarity of the projections means that $P_{\tau}+P_{T}=i d$ and $P_{\tau} P_{T}=$ $P_{T} P_{\tau}=0$ hold. Thus, a form $\omega$ is uniquely decomposed as $\omega=P_{\tau} \omega+P_{T} \omega$. The horizontal component $P_{\tau} \omega=\omega-\tau \wedge i_{T} \omega$, which is the component along symmetry cells defined by $\tau$, is denoted by $\omega_{\tau}$, and the vertical component $P_{T} \omega$ is the component along 
the orbits. If we write $P_{T} \omega$ in terms of the wedge product and contraction, the decomposition reads as

$$
\omega=\omega_{\tau}+\tau \wedge i_{T} \omega
$$

Because $\tau$ is known, the field $\omega$ can be constructed from $\omega_{\tau}$ and $i_{T} \omega$, the geometric components of $\omega$. Thus, the lower-dimensional BVPs are formulated in terms of the geometric components.

Remark 4. The geometric components are of different degree, and only the horizontal component is defined for 0-forms. Similarly, only the vertical component is defined for $m$-forms on an m-manifold. At singular points, $i_{T} \omega=0$ holds because $T=0$ holds. But then because $\tau$ is not defined at singular points, the decomposition of the fields in (1) is not defined either. However, this is not a problem because the singular orbits are assumed to always be at the boundary of the orbit space N. Finally, because $T$ and $\tau$ are G-invariant, the geometric components of a $(G, h)$-invariant form are also $(G, h)$ invariant [[6], p. 117].

\subsection{Horizontal forms}

A differential form $\omega$ is horizontal if $P_{\tau} \omega=\omega$ holds or equivalently if $i_{T} \omega=0$ holds. The geometric components $\omega_{\tau}$ and $i_{T} \omega$ are both horizontal forms. The set $\Omega_{h}^{p}(M)$ of all horizontal $p$-forms on $M$ constitutes a linear subspace of $\Omega^{p}(\mathrm{M})$ and the wedge product of horizontal forms is again a horizontal form [[6], p. 64].

Natural way to transfer differential forms from $M$ to the orbit space $N$ is to use the pullback $\kappa^{*}: \Omega(M) \rightarrow \Omega(N)$ of the cross-section $\kappa$ corresponding to the symmetry cell $A$. The unrestricted $\kappa^{*}$ is not an isomorphism because it is not an injection; however, $\kappa^{*}$ maps bijectively $(G, h)$-invariant horizontal forms on $M$ to differential forms on $N$. Thus, the vector space of all $(G, h)$-invariant horizontal $p$-forms on $M$ is isomorphic to $\Omega^{p}(N)$. If $\omega$ is a solution to a BVP on $M$, the solution to the reduced BVP on $N$ is $\left(\kappa^{*} \omega_{\tau}, \kappa^{*} i_{T} \omega\right)$. To transfer this solution back to $M$ from $N$, we need an inverse for $\kappa^{*}$. Because $\kappa^{*}$ is only a surjection, there exist only right-inverses, denoted by $r$. Thus, $r$ : $\Omega(N) \rightarrow(M)$ is a mapping such that $\kappa^{*} \circ r$ is the identity mapping of $\Omega(N)$. However, because $\kappa^{*}$ is also an injection for $(G, h)$-invariant horizontal forms, there exists such a right-inverse $r$ that $r \circ \kappa^{*}$ is the identity mapping for horizontal forms on $M$, i.e., $r \circ \kappa^{*}$ $=P_{\tau}$ holds.

The observer-induced projections $P_{\tau}$ and $P_{T}$ decompose also the exterior derivative: $d_{M}=P_{\tau} d_{M}+P_{T} d_{M}$. The operator $d_{\tau}=P_{\tau} d_{M}$ is called the horizontal exterior derivative, and it is the exterior derivative of the horizontal forms, because it has all the same characteristics as the exterior derivative [[6], p. 65]. Furthermore, $d_{\tau}$ naturally corresponds to $d_{N}$ via the pullback $\kappa^{*}$ :

$$
\kappa^{*} \circ d_{\tau}=\kappa^{*} \circ P_{\tau} d_{M}=\kappa^{*} \circ d_{M}=d_{N} \circ \kappa^{*} .
$$

Thus, we can make a bijective correspondence between differential equations in the orbit space and differential equations of horizontal forms. At the same time, the vertical exterior derivative $d_{T}=P_{T} d_{M}$ itself is not useful, because $\kappa^{*} d_{T}=0$ holds. However, the useful parts of $d_{T}$ (cf. $P_{T} \omega$ and $i_{T} \omega$ ) can be expressed with the horizontal exterior derivative $d_{\tau}$ and the Lie derivative $\mathcal{L}_{T}$, as shown in the next section. 


\section{Lower-dimensional BVPs for 1-D symmetry groups}

In this section, we formulate the reduced BVPs on the orbit space. We assume that the model BVP is $(G, h)$-invariant under an action of a connected 1-D Lie group $G$ and that the orbit space $N=M / G$ exists. Thus, the boundary values, the constitutive equation, and the source field $Q$ are $(G, h)$-invariant. We also assume that the BVP has unique solution, which implies that the fields $C$ and $K$ are $(G, h)$-invariant (Theorem $2)$. To formulate a reduced BVP, we must choose a $G$-observer $(T, \tau)$. Then, the projections $P_{\tau}$ and $P_{T}$ determine the geometric components. The projections decompose also the differential equations, the boundary values, and the constitutive equation, such that they are expressed in terms of horizontal operators and the geometric components. Thus, the model BVP is decomposed such that when it is pulled back to the orbit space $N$, with the cross-section $\kappa$ corresponding to $\tau$, it can be identified with a BVP in $N$. Finally, to express the decomposed BVP in $N$ in terms of the operators of $N$, we apply various commutation rules of the operators (e.g., Hodge-like operator) with the pullback.

\subsection{Construction of a G-observer and decomposition of fields}

A $G$-observer $(T, \tau)$ is determined uniquely by a selection of a parameterization $\beta: \mathbb{R}$ $\rightarrow G$ (represents the action as a 1-parameter group of transformations) and a symmetry cell $A$ (Def. 11). Notice that the solution to the original BVP does not depend on the choice of G-observer. However, the reduced BVP does depend on the choice, and all choices may not be equally convenient. We shall come back to this subject later on.

The $G$-observer $(T, \tau)$ decomposes the fields $C, K$, and $Q$ according to (1). The fields to be solved from the reduced BVP are the geometric components $C_{\tau}, i_{T} C, K_{\tau}$, and $i_{T} K$. To bijectively identify the geometric components of $C, K$, and $Q$ with fields in the orbit space, we use the pullback of the cross-section $\kappa: N \rightarrow A$.

\subsection{Differential equations of the reduced BVP}

The differential equations in the orbit space are derived from the equations of the model BVP through decomposition by the projections $P_{\tau}$ and $P_{T}$ :

$$
\begin{aligned}
d_{M} C & =Q \\
\Leftrightarrow\left(P_{\tau} d_{M}+P_{T} d_{M}\right)\left(P_{\tau} C+P_{T} C\right) & =P_{\tau} Q+P_{T} Q \\
\Leftrightarrow P_{\tau} d_{M} P_{\tau} C+P_{\tau} d_{M} P_{T} C+P_{T} d_{M} P_{\tau} C+P_{T} d_{M} P_{T} C & =P_{\tau} Q+P_{T} Q .
\end{aligned}
$$

Because $d_{M} \tau=0$ holds for any G-observer $(T, \tau)$ (see Remark 3), we can prove with straightforward calculations and Cartan's formula [2] that the following equations hold for any $\omega[[6], p .68]$ :

$$
\begin{aligned}
& P_{\tau} d_{M} P_{T} \omega=0, \\
& P_{T} d_{M} P_{\tau} \omega=\tau \wedge \mathcal{L}_{T} \omega_{\tau}, \\
& P_{T} d_{M} P_{T} \omega=-\tau \wedge d_{\tau} i_{T} \omega .
\end{aligned}
$$

Then, (3) can be written in terms of the horizontal exterior derivative $d_{\tau}=P_{\tau} d_{M}$, the Lie derivative $\mathcal{L}_{T}$, and the geometric components of $C$ and $Q$ :

$$
d_{\tau} C_{\tau}+\tau \wedge\left(\mathcal{L}_{T} C_{\tau}-d_{\tau} i_{T} C\right),=Q_{\tau}+\tau \wedge i_{T} Q .
$$


Straightforward calculations using the projections $P_{\tau}$ and $P_{T}$ show that (4) is equivalent to the following system of equations:

$$
\left\{\begin{array}{l}
d_{\tau} C_{\tau}=Q_{\tau} \\
d_{\tau} i_{T} C=\mathcal{L}_{T} C_{\tau}-i_{T} Q
\end{array}\right.
$$

Because $C$ is $(G, h)$-invariant, its geometric components are also $(G, h)$-invariant. Thus, $\mathcal{L}_{T} C_{\tau}=h^{\prime}(0) C_{\tau}$ holds by Theorem 1 and substitution of $h^{\prime}(0) C_{\tau}$ for $\mathcal{L}_{T} C_{\tau}$ in the above system of equations yields:

$$
\left\{\begin{array}{l}
d_{\tau} C_{\tau}=Q_{\tau} \\
d_{\tau} i_{T} C=h^{\prime}(0) C_{\tau}+i_{T} Q
\end{array}\right.
$$

The pullback $\kappa^{*}$ bijectively identifies these equations with equations in the orbit space:

$$
\left\{\begin{aligned}
\kappa^{*} d_{\tau} C_{\tau} & =\kappa^{*} Q_{\tau} \\
\kappa^{*} d_{\tau} i_{T} C & =h^{\prime}(0) \kappa^{*} C_{\tau}+\kappa^{*} i_{T} Q .
\end{aligned}\right.
$$

The equations are still expressed in terms of $d_{\tau}$. However, $\kappa^{*} \circ d_{\tau}=d_{N} \circ \kappa^{*}$ holds by (2), and thus, we get the desired equations that are expressed in terms of $d_{N}$ :

$$
\begin{aligned}
& d_{N}\left(\kappa^{*} C_{\tau}\right)=\kappa^{*} Q_{\tau}, \\
& d_{N}\left(\kappa^{*} i_{T} C\right)=h^{\prime}(0) \kappa^{*} C_{\tau}+\kappa^{*} i_{T} Q .
\end{aligned}
$$

Similar derivation applied to the other differential equation in the model BVP produces the following equations:

$$
\begin{aligned}
& d_{N}\left(\kappa^{*} K_{\tau}\right)=0, \\
& d_{N}\left(\kappa^{*} i_{T} K\right)=h^{\prime}(0) \kappa^{*} K_{\tau} .
\end{aligned}
$$

Equations 5-8 are the differential equations on the orbit space for the reduced BVP. Interestingly, some of the equations may hold trivially: for example, if the degree of $\kappa^{*} K_{\tau}$ is the same as the dimension of $N$, then its exterior derivative is always zero. The fields governed by these trivial equations are solved by substitution into the constitutive equations.

Example 1. Let the model BVP depict a magnetostatic BVP. If the BVP is G-invariant $\left(h^{\prime}(0)=0\right)$, then the differential equations for the reduced BVP in the 2-D orbit space $N$ are

$$
\left\{\begin{array}{l}
d_{N}\left(\kappa^{*} i_{T} B\right)=0 \\
d_{N}\left(\kappa^{*} H_{\tau}\right)=\kappa^{*} J_{\tau} \\
d_{N}\left(\kappa^{*} i_{T} H\right)=\kappa^{*} i_{T} J \\
d_{N}\left(\kappa^{*} B_{\tau}\right)=0 .
\end{array}\right.
$$

The last equation is a trivial equation. Notice that only G-invariance of current $J$ is assumed, but not any special direction. Often, only one of the geometric components of J is assumed to be nonzero, in which case the current is either in the direction of the orbits or is purely horizontal. This makes one of the equations for $H$ homogeneous and often very easy to solve. $\square$ 


\subsection{Boundary values of the reduced BVP}

The $(G, h)$-invariant boundary values of the Model BVP can be decomposed with the observer-induced projections. Then, the pullback $\kappa^{*}$ bijectively identifies these decomposed boundary values with boundary values in the orbit space. Singular orbits always reside, by assumption, on the boundary $\partial N$ of the orbit space. For those singular orbits whose points are not part of the boundary $\partial M$, the symmetry itself will induce the boundary values.

The boundary $\partial N$ is a union $\partial N=S \cup E$, where $S$ is the set of all singular orbits whose points are not included in $\partial M$, and $E$ is the part of $\partial N$ that can be embedded into $\partial M$ with a cross-section (see Figure 5).

Furthermore, in the model BVP, the boundary $\partial M$ consists of two complementary parts $\left(\partial M=\partial M^{1} \cup \partial M^{2}\right)$, and therefore, also $E$ consists of two complementary parts, or $E=E^{1} \cup E^{2}$ holds.

To derive the boundary values at $E$, we need the restrictions $P_{t \tau}=i_{t T} I_{t \tau}$ and $P_{t T}=I_{t \tau}$ $i_{t T}$ of the projections $P_{\tau}$ and $P_{T}$ to the boundary $\partial M$ ( $t \tau$ and $t T$ denote the restrictions of $\tau$ and $T$ to $\partial M)$. Then, with these projections, we can decompose the boundary values of the model BVP:

$$
\begin{aligned}
t_{\partial M^{1}} C & =c \\
\Leftrightarrow t_{\partial M^{1}} P_{\tau} C+t_{\partial M^{1}} P_{T} C & =P_{t \tau} c+P_{t T} c .
\end{aligned}
$$

Then, because $P_{t \tau}$ and $P_{t T}$ are complementary and because $t_{\partial M} \circ P_{\tau}=P_{t \tau} \circ t_{\partial M}$ and $t_{\partial M} \circ P_{T}=P_{t T} \circ t_{\partial M}$ hold, Equation 9 is equivalent to the following system of equations:

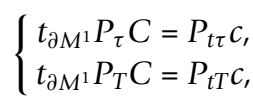

which can be written as follows:

$$
t_{\partial M^{1}} C_{\tau}=c_{\tau}
$$

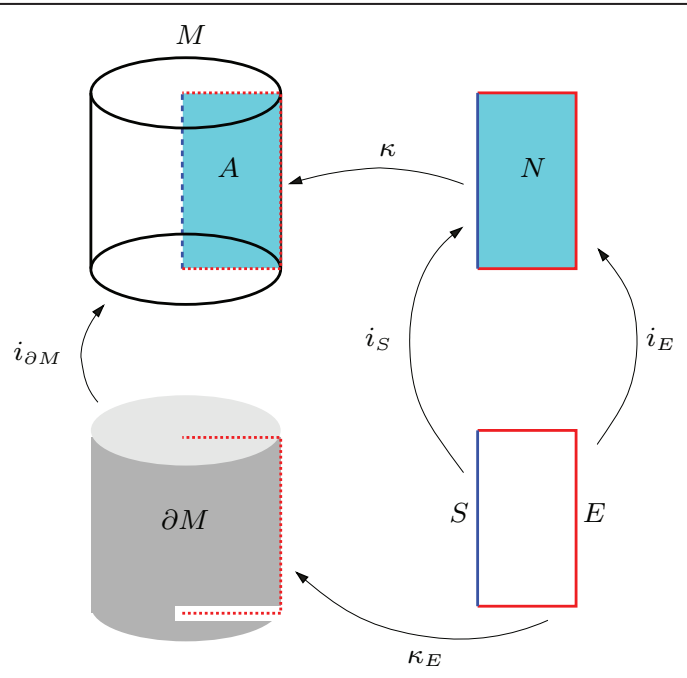

Figure 5 Embedding the boundary of the orbit space. $M$ is a 3-manifold with rotational symmetry. $A$ is a symmetry cell, i.e., the image of the orbit space $N$ under a cross-section $\kappa$. The boundary $\mathrm{ON}$ consists of parts $E$ and $S$ whose points correspond to the points in $\partial M$ and in the axis of rotation, respectively. $i_{E}$ and $i_{S}$ are the inclusion maps of $E$ and $S$ into $N$, and $\kappa_{E}$ embeds $E$ into $\partial M$. 


$$
t_{\partial M^{1}} I_{\tau} i_{T} C=I_{t \tau} i_{t T} C
$$

Because of the relation $\tau(T)=1$,

$$
I_{\tau} i_{T} \omega=I_{\tau} i_{T} \eta \Leftrightarrow i_{T} \omega=i_{T} \eta
$$

holds for all forms $\omega$ and $\eta$. This fact together with the commutations $t \partial M \circ I_{\tau}=I_{t \tau}$ $\circ t_{\partial M}$ and $t_{\partial M} \circ i_{T}=i_{t T} \circ t_{\partial M}$ makes Equation 11 equivalent to the following:

$$
t_{\partial M^{1}} i_{T} C=i_{t T} c
$$

Equations 10 and 13 express the equation $t_{\partial M}^{1} C=c$ in terms of the geometric components. To identify them bijectively with equations in $N$, we use the pullback of the embedding $\kappa_{E}: E \rightarrow \partial M$ : If $i_{E}$ and $i_{\partial M}$ are the inclusion maps of the boundaries $E$ and $\partial M$ to $N$ and $M$, respectively, then $\kappa_{E}$ is defined such that

$$
i_{\partial M} \circ \kappa_{E}=\kappa \circ i_{E}
$$

holds (see Figure 5). Pullbacks to the composite mappings of Equation 14 satisfy the following commutation rule:

$$
\kappa_{E}^{*} \circ t_{\partial M}=t_{E} \circ \kappa^{*} \text {. }
$$

Then, just operation by $\kappa_{E}^{*}$ to Equations 10 and 13 will give the boundary values for the fields $\kappa^{*} C_{\tau}$ and $\kappa^{*} i_{T} C$ :

$$
\begin{aligned}
& t_{E^{1}}\left(\kappa^{*} C_{\tau}\right)=\kappa_{E^{1}}^{*} C_{\tau}, \\
& t_{E^{1}}\left(\kappa^{*} i_{T} C\right)=\kappa_{E^{1}}^{*} i_{T} c .
\end{aligned}
$$

Similar derivation for fields $\kappa^{*} K_{\tau}$ and $\kappa^{*} i_{T} K$ yields:

$$
\begin{aligned}
& t_{E^{2}}\left(\kappa^{*} K_{\tau}\right)=\kappa_{E^{2}}^{*} k_{\tau}, \\
& t_{E^{2}}\left(\kappa^{*} i_{T} K\right)=\kappa_{E^{2}}^{*} i_{T} k .
\end{aligned}
$$

At singular points, the vector field $T$ is always the zero vector, and therefore, $i_{T} \omega=0$ holds on them. Thus, the symmetry fixes the fields $\kappa^{*} i_{T} C$ and $\kappa^{*} i_{T} K$ to zero at the singular orbits:

$$
\begin{aligned}
& t_{s}\left(\kappa^{*} i_{T} C\right)=0, \\
& t_{s}\left(\kappa^{*} i_{T} K\right)=0,
\end{aligned}
$$

where $t_{S}$ denotes the pullback of the inclusion map $i_{S}: S \rightarrow \partial N$ (see Figure 5 ). On the other hand, the fields $\kappa^{*} C_{\tau}$ and $\kappa^{*} K_{\tau}$ are not even defined at singular orbits (see Remark 4). However, the fields $C$ and $K$ can still be solved for and they need not be zero at singular interior points of $M$, which can be easily seen from the following magnetostatic BVP: The domain is the one shown in Figure 4, and there is a static current in the circular conductor. Clearly, the magnetic field $H$ and flux density $B$ are not zero at the points of the rotational axis, but nonzero and aligned along the direction of the axis. Because the fields $H$ and $B$ are smooth in small neighborhoods of the axis points, we can uniquely extend the solution for these points. 
Equations 15-20 are the boundary values on the orbit space for the reduced BVP. Observe that non-homogeneous boundary conditions on $\partial M$ may induce homogeneous conditions on $\partial N$, because $\partial N$ is an $(n-2)$-manifold and all forms of degree $(n-1)$ or $n$ are always zero at $\partial N$. However, there is no contradiction, because the contracted forms, e.g., $i_{T} k$, need not be zero. Furthermore, these homogeneous equations hold trivially, they always correspond to the fields that also have trivial differential equations, and they are solved for by substitution into the constitutive equations.

\subsection{Constitutive equations of the reduced BVP}

The constitutive equation gets decomposed by the observer-induced projections, and this induces a decomposition of the Hodge-like operator $v$ into four linear operators. When the decomposition of the constitutive equation is pulled back to the orbit space with $\kappa^{*}$, we get the constitutive equations for the fields $\kappa^{*} i_{T} K$ and $\kappa^{*} K_{\tau}$ in terms of the fields $\kappa^{*} C_{\tau}$ and $\kappa^{*} i_{T} C$.

The projections decompose the constitutive equation of the model BVP:

$$
\begin{aligned}
K & =v C \\
\Leftrightarrow P_{\tau} K+P_{T} K & =v P_{\tau} C+v P_{T} C \\
\Leftrightarrow P_{\tau} K+P_{T} K & =P_{\tau} v P_{\tau} C+P_{T} v P_{\tau} C+P_{\tau} v P_{T} C+P_{T} v P_{T} C .
\end{aligned}
$$

Because $v$ is a bijection and the projections are complementary, the equation above is equivalent to the following system of equations:

$$
\left\{\begin{array}{l}
P_{\tau} K=P_{\tau} v P_{\tau} C+P_{\tau} v P_{T} C \\
P_{T} K=P_{T} v P_{\tau} C+P_{T} v P_{T} C .
\end{array}\right.
$$

To express the constitutive equation in terms of the geometric components of the fields $C$ and $K$, we use Equation 12:

$$
\left\{\begin{aligned}
K_{\tau} & =\left(P_{\tau} v\right)\left(C_{\tau}\right)+\left(P_{\tau} v I_{\tau}\right)\left(i_{T} C\right) \\
i_{T} K & =\left(i_{T} v\right)\left(C_{\tau}\right)+\left(i_{T} v I_{\tau}\right)\left(i_{T} C\right)
\end{aligned}\right.
$$

This shows the decomposition of $v$ into four linear operators that map horizontal forms to horizontal forms.

Let us next pull back these equations into the orbit space with $\kappa^{*}$ and use the property $\kappa^{*} P_{\tau}=\kappa^{*}$ :

$$
\begin{aligned}
& \kappa^{*} K_{\tau}=\left(\kappa^{*} v\right)\left(C_{\tau}\right)+\left(\kappa^{*} v I_{\tau}\right)\left(i_{T} C\right), \\
& \kappa^{*} i_{T} K=\left(\kappa^{*} i_{T} v\right)\left(C_{\tau}\right)+\left(\kappa^{*} i_{T} v I_{\tau}\right)\left(i_{T} C\right) .
\end{aligned}
$$

To express these equations in terms of fields $\kappa^{*} C_{\tau}$ and $\kappa^{*} i_{T} C$, we define the linear operators $v_{\tau}^{\tau}, v_{T}^{\tau}, v_{\tau}^{T}$, and $v_{T}^{T}$ that map from $\Omega(N)$ to itself such that they satisfy the following commutation rules for all horizontal forms on $M$ :

$$
\begin{aligned}
\kappa^{*} \circ v & =v_{\tau}^{\tau} \circ \kappa^{*}, \\
\kappa^{*} \circ v \circ I_{\tau} & =v_{T}^{\tau} \circ \kappa^{*}, \\
\kappa^{*} \circ i_{T} \circ v & =v_{\tau}^{T} \circ \kappa^{*}, \\
\kappa^{*} \circ i_{T} \circ v \circ I_{\tau} & =v_{T}^{T} \circ \kappa^{*} .
\end{aligned}
$$


The operators can be given explicitly with a right-inverse $r$ of $\kappa^{*}$ that satisfies $r \circ \kappa^{*}$ $=P_{\tau}($ see Section 7.3):

$$
\begin{aligned}
& v_{\tau}^{\tau}=\kappa_{m-p}^{*} \circ v \circ r_{p,} \\
& v_{T}^{\tau}=\kappa_{m-p}^{*} \circ v \circ I_{\tau} \circ r_{p-1}, \\
& v_{\tau}^{T}=\kappa_{m-p-1}^{*} \circ i_{T} \circ v \circ r_{p \prime} \\
& v_{T}^{T}=\kappa_{m-p-1}^{*} \circ i_{T} \circ v \circ I_{\tau} \circ r_{p-1},
\end{aligned}
$$

where the subindices in $\kappa^{*}$ and $r$ indicate the degree of the form they operate on. With these operators, Equations 21 and 22 become

$$
\begin{aligned}
& \kappa^{*} K_{\tau}=v_{\tau}^{\tau}\left(\kappa^{*} C_{\tau}\right)+v_{T}^{\tau}\left(\kappa^{*} i_{T} C\right), \\
& \kappa^{*} i_{T} K=v_{\tau}^{T}\left(\kappa^{*} C_{\tau}\right)+v_{T}^{T}\left(\kappa^{*} i_{T} C\right) .
\end{aligned}
$$

Equations 23 and 24 are the constitutive equations on the orbit space for the reduced BVP. They hold at the interior points of $N$.

Example 2. Let us present the matrices of the operators $v_{\tau}^{\tau}, v_{\tau}^{\tau}$, $v_{T}^{\tau}$, and $v_{T}^{T}$ bases that are compatible with the G-observer. Let $C$ and $K$ be 1- and 2-forms, respectively, on a 3-manifold $M$. Let $X, Y$, and $Z$ be G-invariant vector fields on $M$ such that $(X, Y, Z)$ forms a basis field, where $(X, Y)$ is a basis field for a chosen symmetry cell $A$ and $Z$ is in the direction of the orbits. Then, the dual basis $(d x, d y, d z)$ is a basis for 1-forms, and $(Z, d z)$ is a G-observer compatible with the basis fields $(X, Y, Z)$. Furthermore, let $(d y \wedge d z, d z \wedge d x, d x \wedge d y)$ be the basis for 2-forms. Then, there exist component 0forms $C_{x}, C_{y}, C_{z} K_{x}, K_{y}$ and $K_{z}$ such that in these bases, $C=C_{x} d x+C_{y} d y+C_{z} d z$ and $K=K_{x} d y \wedge d_{z}+K_{y} d z \wedge d x+K_{z} d x \wedge d y$ hold. The geometric components $C_{d z} i_{Z} C, K_{d z}$ and $i_{Z} K$ in terms of the above bases are

$$
\begin{aligned}
& C_{d z}=C_{x} d x+C_{y} d y, \\
& i_{Z} C=C_{z \prime} \\
& K_{d z}=K_{z} d x \wedge d y, \\
& i_{Z} K=K_{y} d x-K_{x} d y .
\end{aligned}
$$

The pullback $\kappa^{*}$ brings the above bases of 1- and 2-forms to corresponding bases of 1and 2-forms into the orbit space $N$. In these pullback bases, the component 0-forms of the geometric components in $N$ are the same as in $M$. If in the above-defined bases, the operator $v$ is expressed as the matrix

$$
v=\left[\begin{array}{lll}
v_{x x} & v_{x y} & v_{x z} \\
v_{y x} & v_{y y} & v_{y z} \\
v_{z x} & v_{z y} & v_{z z}
\end{array}\right],
$$

then the matrices of the linear operators $v_{d z}^{d z}, v_{Z}^{d z}, v_{d z}^{Z}$, and $v_{Z}^{Z}$ in the pullback bases are

$$
\begin{gathered}
v_{d z}^{d z}=\left[\begin{array}{ll}
v_{z x} & v_{z y}
\end{array}\right], \quad v_{Z}^{d z}=\left[v_{z z}\right], \\
v_{d z}^{Z}=\left[\begin{array}{cc}
v_{y x} & v_{y y} \\
-v_{x x} & v_{x y}
\end{array}\right], \quad v_{Z}^{Z}=\left[\begin{array}{l}
v_{y z} \\
-v_{x z}
\end{array}\right] .
\end{gathered}
$$




\subsection{The reduced BVP and the choice of G-observer}

The reduced model BVP on the orbit space $N$, corresponding to a G-observer $(T, \tau)$, consists of the differential equations (5)-(8), the boundary values (15) - (20), and the constitutive equations (23) - (24):

$$
\begin{aligned}
& d_{N}\left(\kappa^{*} C_{\tau}\right)=\kappa^{*} Q_{\tau}, \quad t_{E^{1}}\left(\kappa^{*} C_{\tau}\right)=\kappa_{E^{1}}^{*} C_{\tau}, \\
& d_{N}\left(\kappa^{*} i_{T} C\right)=h^{\prime}(0) \kappa^{*} C_{\tau}+\kappa^{*} i_{T} Q, \quad t_{E^{1}}\left(\kappa^{*} i_{T} C\right)=\kappa_{E^{1}}^{*} i_{T} c, \\
& d_{N}\left(\kappa^{*} K_{\tau}\right)=0 \text {, } \\
& d_{N}\left(\kappa^{*} i_{T} K\right)=h^{\prime}(0) \kappa^{*} K_{\tau} \text {, } \\
& t_{E^{2}}\left(\kappa^{*} K_{\tau}\right)=\kappa_{E^{2}}^{*} k_{\tau} \text {, } \\
& t_{E^{2}}\left(\kappa^{*} i_{T} K\right)=\kappa_{E^{2}}^{*} i_{T} k \text {, } \\
& t_{s}\left(\kappa^{*} i_{T} C\right)=0 \text {, } \\
& \kappa^{*} K_{\tau}=v_{\tau}^{\tau}\left(\kappa^{*} C_{\tau}\right)+v_{T}^{\tau}\left(\kappa^{*} i_{T} C\right), \\
& t_{s}\left(\kappa^{*} i_{T} K\right)=0 \text {, } \\
& \kappa^{*} i_{T} K=v_{\tau}^{T}\left(\kappa^{*} C_{\tau}\right)+v_{T}^{T}\left(\kappa^{*} i_{T} C\right) \text {. }
\end{aligned}
$$

These equations show that in the most general case, the terms $h^{\prime}(0) \kappa^{*} C_{\tau}$ and $h^{\prime}(0)$ $\kappa^{*} K_{\tau}$ in the differential equations and the operators $v_{\tau}^{\tau}$ and $v_{T}^{T}$ in the constitutive equations couple the system of four differential equations.

Let us examine when the equations decouple. First, if the model BVP is G-invariant $\left(h^{\prime}(0)=0\right)$ and if the operators $v_{\tau}^{\tau}$ and $v_{T}^{T}$ are zero, then reduced BVP breaks up into two decoupled BVPs:

$$
\begin{aligned}
& d_{N}\left(\kappa^{*} C_{\tau}\right)=\kappa^{*} Q_{\tau}, \quad t_{E^{1}}\left(\kappa^{*} C_{\tau}\right)=\kappa_{E 1}^{*} C_{\tau}, \\
& \kappa^{*} i_{T} K=v_{\tau}^{T}\left(\kappa^{*} C_{\tau}\right), t_{E^{2}}\left(\kappa^{*} i_{T} K\right)=\kappa_{E^{2}}^{*} i_{T} k, \\
& d_{N}\left(\kappa^{*} i_{T} K\right)=0, \quad t_{s}\left(\kappa^{*} i_{T} K\right)=0, \\
& d_{N}\left(\kappa^{*} i_{T} C\right)=\kappa^{*} i_{T} Q, \quad t_{E^{1}}\left(\kappa^{*} i_{T} C\right)=\kappa_{E^{1}}^{*} i_{T} C, \\
& d_{N}\left(\kappa^{*} K_{\tau}\right)=0, \quad \begin{aligned}
t_{\mathrm{s}}\left(\kappa^{*} i_{T} C\right) & =0 .
\end{aligned}
\end{aligned}
$$

In the case of G-invariant BVP, the decoupling can be achieved also without $v_{\tau}^{\tau}$ and $v_{T}^{T}$ being zero, if one of the geometric components is zero: If, for example, $d_{N}\left(\kappa^{*} K_{\tau}\right)=$ 0 is a trivial equation, in which case the boundary value $\kappa_{E^{2}}^{*} k_{\tau}$ is zero, and if the source $\kappa^{*} i_{T} Q$ and the boundary value $\kappa_{E^{1}}^{*} i_{T} \mathcal{C}$ are zero, then $\kappa^{*} i_{T} C$ must be zero. In this way, the coupling via constitutive equations disappears, and we have the following BVP to solve:

$$
\begin{array}{lll}
d_{N}\left(\kappa^{*} C_{\tau}\right)=\kappa^{*} Q_{\tau}, & \kappa^{*} i_{T} K=v_{\tau}^{T}\left(\kappa^{*} C_{\tau}\right), & t_{E^{1}}\left(\kappa^{*} C_{\tau}\right)=\kappa_{E^{1}}^{*} C_{\tau}, \\
d_{N}\left(\kappa^{*} i_{T} K\right)=0, & \kappa^{*} K_{\tau}=v_{\tau}^{\tau}\left(\kappa^{*} C_{\tau}\right), & \\
& t_{s}\left(\kappa^{*} i_{T} K\right)=\kappa_{E^{2}}^{*} i_{T} k,
\end{array}
$$

Observe that the equation $\kappa^{*} K_{\tau}=v_{\tau}^{\tau}\left(\kappa^{*} C_{\tau}\right)$ is for evaluation only.

Because the operators $v_{\tau}^{\tau}$ and $v_{T}^{T}$ depend on the G-observer $(T, \tau)$, their being zero may depend on the choice of the G-observer. Let us next examine when it is possible to choose an observer that makes the operators $v_{\tau}^{\tau}$ and $v_{T}^{T}$ zero. The operators are zero if $v, T$, and $\tau$ are such that $i_{T}(v(\tau \wedge \omega))=0$ holds for all $\omega \in \Omega^{p-1}(M)$. Let us study what this requirement means geometrically, and for simplicity, we take $v_{M}: \Omega^{1}(M) \rightarrow$ $\Omega^{m-1}(M)$, in which case $\omega$ is a 0 -form and can be ignored. Thus, we have $i_{T}(v(\tau))=0$, and by the Def. 2 , this is equivalent to $\left(i_{T} v_{\varphi} \star_{\varphi}\right)(\tau)=0$, where $\varphi$ is some metric tensor on $M$. Now, if $\varphi$ is such that $v_{\varphi}$ is a scalar field $v_{0}$, we have $\left(v_{0} i_{T^{\star}}\right)(\tau)=0$. Then, by the Definition of Hodge-operator, this is equivalent to $v_{0} i_{T} i_{\# \tau} v o l=0$, where $\sharp \tau$ is the 
metric dual [4] of $\tau$ and vol the volume element [2] of $\varphi$. This equation means that $\# \tau$ and $T$ are parallel and the symmetry cell is everywhere orthogonal to $T$. Thus, the operators $v_{\tau}^{\tau}$ and $v_{T}^{T}$ to be zero, there must be such a metric $\varphi$ that $v_{\varphi}$ is a scalar field and the orbits are everywhere orthogonal to the symmetry cell.

\section{Multi-dimensional symmetry groups}

In this section, we formulate reduced BVPs with multi-dimensional symmetry groups $G$ that are products of connected 1-D Lie groups. This may sound like a restriction, but for instance, all connected 2-D Lie groups are products of 1-D Lie groups [13]. Moreover, in 3-D symmetries, where time is one of the dimensions, the Lie groups are also always products of 1-D Lie groups. When $G$ is a product of 1-D groups, we can apply the previous results: The group action is a composition of the separate actions of the 1-D factor groups, and we can iteratively apply each factor group one at a time and use the results of the previous sections. We can also make a direct approach where we apply all the factors at once and deduce the lower-dimensional BVP directly. A more detailed exposition can be found in [6].

\subsection{Iterative approach}

Assume that the symmetry group $G=G_{1} \times \cdots \times G_{n}$ is a product of $n \leq m$ 1-D Lie groups $G_{i}$. Furthermore, assume that the group actions $F_{i}: G_{i} \times M \rightarrow M$ are separate from every other $F_{j}, j \neq i$. Finally, assume that the symmetry transformations of the group actions $F_{i}$ commute, i.e., $g_{i} \circ g_{j}=g_{j} \circ g_{i}$ holds for all $g_{i} \in G_{i}, g_{j} \in G_{j}$.

Let $G_{1}$ act on $M$. Then, with a $G_{1}$-observer, we can formulate a lower-dimensional BVP on the orbit space $M / G_{1}$. Now, the group action $F_{2}$ of $G_{2}$ on $M$ induces a group action $F_{2}^{1}$ on $M / G_{1}$ such that $F_{2}^{1}\left(g_{2}, G_{1} x\right)=G_{1} F_{2}\left(g_{2}, x\right)$ holds, where $G_{1} x$ and $G_{1} F_{2}\left(g_{2}\right.$, $x)$ are the orbits of points $x, F_{2}\left(g_{2}, x\right) \in M$ under the action $F_{1}$. For example, if $F_{1}$ and $F_{2}$ consist of translations and rotations, respectively, then the relation $F_{2}^{1}\left(g_{2}, G_{1} x\right)=G_{1} F_{2}\left(g_{2}, x\right)$ means the following: The translation of point $x \in M$ forms the orbit $G_{1} x \in M / G_{1}$, and then $G_{1} x$ is rotated by $F_{2}^{1}$ to the orbit $G_{1} F_{2}\left(g_{2}, x\right)$ containing the point $F_{2}\left(g_{2}, x\right)$, which is the point where $x$ is rotated by $F 2$. The action $F_{2}^{1}$ now has its own orbit space $\left(M / G_{1}\right) / G_{2}$, and by choosing a $G_{2}$-observer on $M / G_{1}$, we can apply the dimensional reduction. The process continues similarly down to the last group $G_{n}$.

\subsection{Direct approach}

From the three starting assumptions of the iterative approach, it follows that the composition $F=F_{1} \circ \ldots \circ F_{n}$ is a group action of $G$ on $M$. Now, we construct $G_{i}$-observers $\left(\begin{array}{ll}T_{i} & \tau_{i}\end{array}\right)$ on $M . T_{i}$ are $G_{i}$-invariant vector fields induced by the actions $F_{i}$ represented as 1-parameter groups of transformations. Then, to define 1-forms $\tau_{i}$, we select a symmetry cell $A$ of the action $F(A$ is an $(m-n)$-dimensional submanifold of $M)$. For the identity $e_{i}$ of $G_{i}$, the image $A i=F\left(G_{1}, \ldots, G_{i-1}, e_{i}, G_{i+1}, \cdots, G_{n}, A\right)$ of $A$ is a symmetry cell of the action $F_{i}$. Then, let $\tau_{i}$ be $G$-invariant 1 -forms such that $\tau_{i}\left(T_{i}\right)=1$ and $\tau_{i}\left(T_{j}\right)=0$ $(i \neq j)$ hold everywhere and if $v$ is tangent to $A$, then $\tau_{i}(v)=0$ holds. Now, $\left(T_{i}, \tau_{i}\right)$ are the $G_{i}$-observers, and together, they form a $G$-observer.

Each pair $\left(T_{i}, \tau_{i}\right)$ of a G-observer define s a pair of complementary projections $P_{\tau_{i}}$ and $P_{T_{i}}$ on $\Omega(M)$. Then, we can decompose fields, differential equations, boundary values, and constitutive equations by applying the projections of the $G$-observer just like in 
Section 8. For example, if $\{(T, \tau) ;(Z, \zeta)\}$ is a $G$-observer, then a field $K$ is decomposed as follows:

$$
\begin{aligned}
K & =P_{\tau} K+P_{T} K \\
& =P_{\zeta} P_{\tau} K+P_{Z} P_{\tau} K+P_{\zeta} P_{T} K+P_{Z} P_{T} K \\
& =\left(K_{\tau}\right)_{\zeta}+\zeta \wedge\left(i_{Z} K_{\tau}\right)+\tau \wedge\left(i_{T} K\right)_{\zeta}+\tau \wedge \zeta \wedge i_{z}\left(i_{T} K\right) .
\end{aligned}
$$

The geometric components of $K$ are $\left(K_{\tau}\right)_{\zeta}, i_{Z} K_{\tau},\left(i_{T} K\right)_{\zeta}$, and $i_{Z}\left(i_{T} K\right)$. The geometric components are horizontal forms because the projection $P_{\zeta} P_{\tau}$ is an identity mapping for them.

\section{Dimensional reduction theorem}

In this section, we give sufficient conditions for a linear BVP described in the model BVP to be solvable as a lower-dimensional BVP. The conditions are precise, and they constitute a systematic checklist, that, when confirmed by the modeler, will guarantee that the BVP can be solved as a lower-dimensional BVP.

Theorem 3. (Dimensional reduction theorem) Let a linear BVP on m-manifold $M$ described in the model BVP have a unique solution, and additionally, let $G$ be an ndimensional $(n \leq m)$ Lie group that is a product of 1-D connected Lie groups and $h: G \rightarrow \mathbb{F}$ be a differentiable homomorphism. If $G$ acts effectively on $M$ such that

(1) the symmetry transformations of the action are diffeomorphisms, and

(2) the source and boundary values are $(G, h)$-invariant and the Hodge-like operator and the cohomology conditions are $(G, h)$-invariance-preserving, and

(3) the orbit space exists, and

(4) all the singular orbits reside at the boundary of the orbit space, and

(5) aG-observer exists,

then the BVP can be equivalently stated as an $(m-n)$-dimensional BVP on the orbit space with a unique solution.

Proof. The assumptions (1) and (2) together with Theorem 2 show that the solution fields $C$ and $K$ are $(G, h)$-invariant. Then, based on the assumptions (3)-(5), we have shown in Sections 8 and 9 how to constructively formulate the $(m-n)$-dimensional BVP on the orbit space corresponding to the chosen G-observer. The pullback of the unique solution of the model BVP to the orbit space is a solution for the reduced BVP. Thus, a solution exists for the reduced BVP. On the other hand, every solution of the reduced BVP induces also a solution for the model BVP by $(G, h)$-invariance of the fields. However, because of the unique solution of the model BVP, the induced solutions must be the same and hence same also in the reduced BVP. Thus, the reduced BVP has a unique solution. $\square$

\section{Examples}

We give two detailed examples of the use of dimensional reduction in problems of electromagnetism. The solutions of these problems are well known, but usually, they are not recognized explicitly to based on symmetry, and they are obtained by working directly with coordinates. Notice also that the static and time-harmonic Maxwell's 
equations are the reduced differential equations of Maxwell's equations in spacetime [8] under an $\mathbb{R}$-invariance and a harmonic $(\mathbb{R}, h)$-invariance w.r.t. time [6].

\subsection{Modes of a waveguide}

Waveguides have uniform cross-sections and are infinitely long. Furthermore, timeharmonic invariance of fields is assumed. Under these assumptions, modes of EM waves arise, and they can be analyzed in the 2-D cross-sections of the waveguide. In other words, the symmetry group $G$ is $(\mathbb{R},+)$, and the boundary values, the Hodge-like operators $\varepsilon$ and $\mu$, and the fields $E, D, H$, and $B$ are all $(G, h)$-invariant.

In the 3-D domain manifold $M$ (corresponds to the waveguide), where there are no free currents or charges and the waveguide is filled with dielectric material, the following time-harmonic Maxwell's equations and constitutive equations hold:

$$
\begin{aligned}
& d_{M} D=0, \quad B=\mu H, \\
& d_{M} H=j \omega D, \quad D=\varepsilon E . \\
& d_{M} B=0, \\
& d_{M} E=-j \omega B,
\end{aligned}
$$

Let us next choose a $G$-observer $(Z, \zeta)$, where $Z$ is in the direction of the orbits, i.e., in the direction of the waveguide and $\zeta$ corresponds to the cross-section $\kappa$, i.e., to some cross-section of the waveguide. The fields are assumed to be $(G, h)$-invariant with $h=e^{-\gamma g}$ (in which case $h^{\prime}(0)=-\gamma$ holds), where $\gamma$ is the complex propagation constant in the direction of the orbits. Then, the above time-harmonic Maxwell's equations are decomposed by the observer into eight equations in the 2-D orbit space $N$, but only the following six equations are nontrivial:

$$
\begin{aligned}
& d_{N}\left(\kappa^{*} i_{Z} D\right)=-\gamma \kappa^{*} D_{\zeta}, \\
& d_{N}\left(\kappa^{*} H_{\zeta}\right)=j \omega\left(\kappa^{*} D_{\zeta}\right), \\
& d_{N}\left(\kappa^{*} i_{Z} H\right)=-\gamma \kappa^{*} H_{\zeta}+j \omega\left(\kappa^{*} i_{Z} D\right), \\
& d_{N}\left(\kappa^{*} i_{Z} B\right)=-\gamma \kappa^{*} B_{\zeta}, \\
& d_{N}\left(\kappa^{*} E_{\zeta}\right)=-j \omega\left(\kappa^{*} B_{\zeta}\right), \\
& d_{N}\left(\kappa^{*} i_{Z} E\right)=-\gamma \kappa^{*} E_{\zeta}-j \omega\left(\kappa^{*} i_{Z} B\right) .
\end{aligned}
$$

The constitutive equations in the orbit space are as follows:

$$
\begin{aligned}
& \kappa^{*} B_{\zeta}=\mu_{\zeta}^{\zeta}\left(\kappa^{*} H_{\zeta}\right)+\mu_{Z}^{\zeta}\left(\kappa^{*} i_{Z} H\right), \\
& \kappa^{*} i_{Z} B=\mu_{\zeta}^{Z}\left(\kappa^{*} H_{\zeta}\right)+\mu_{Z}^{Z}\left(\kappa^{*} i_{Z} H\right), \\
& \kappa^{*} D_{\zeta}=\varepsilon_{\zeta}^{\zeta}\left(\kappa^{*} E_{\zeta}\right)+\varepsilon_{Z}^{\zeta}\left(\kappa^{*} i_{Z} E\right), \\
& \kappa^{*} i_{Z} D=\varepsilon_{\zeta}^{Z}\left(\kappa^{*} E_{\zeta}\right)+\varepsilon_{Z}^{Z}\left(\kappa^{*} i_{Z} E\right) .
\end{aligned}
$$


Together with the boundary values, Equations 25-34 completely define the electromagnetic fields in a waveguide.

In the case of $T E$-modes of a rectangular waveguide, we assume that $i_{Z} E=0$ holds. Furthermore, if the materials are appropriate (e.g., isotropic) and the $G$-observer is selected conveniently, then we can make $\mu_{\zeta}^{\zeta}=\varepsilon_{\zeta}^{\zeta}=0$ and $\mu_{Z}^{Z}=\varepsilon_{Z}^{Z}=0$ hold. Thus, the differential equations on the orbit space in terms of the geometric components of $E$ and $H$ are:

$$
\begin{aligned}
& d_{N} \varepsilon_{\zeta}^{Z}\left(\kappa^{*} E_{\zeta}\right)=0, \\
& d_{N}\left(\kappa^{*} H_{\zeta}\right)=0, \\
& d_{N}\left(\kappa^{*} i_{Z} H\right)=-\gamma \kappa^{*} H_{\zeta}+j \omega \varepsilon_{\zeta}^{Z}\left(\kappa^{*} E_{\zeta}\right), \\
& d_{N} \mu_{Z}^{\zeta}\left(\kappa^{*} H_{\zeta}\right)=-\gamma \mu_{Z}^{\zeta}\left(\kappa^{*} i_{Z} H\right), \\
& d_{N}\left(\kappa^{*} E_{\zeta}\right)=-j \omega \mu_{Z}^{\zeta}\left(\kappa^{*} i_{Z} H\right), \\
& \gamma \kappa^{*} E_{\zeta}=-j \omega \mu_{Z}^{\zeta}\left(\kappa^{*} H_{\zeta}\right) .
\end{aligned}
$$

From (37), (38), and (40), we can derive the wave equation for $\kappa^{*} i_{z} H$ :

$$
\left(\mu_{Z}^{\zeta}\right)^{-1} d_{N} \mu_{\zeta}^{z} d_{N}\left(\kappa^{*} i_{Z} H\right)+\left(\mu_{Z}^{\zeta}\right)^{-1} h^{2} \mu_{Z}^{\zeta}\left(\kappa^{*} i_{Z} H\right)=0,
$$

where $h^{2}=-\gamma^{2}+\omega^{2} \varepsilon_{\zeta}^{Z} \mu_{\zeta}^{Z}$ holds. In a suitable $x y$-coordinate system, the above wave equation is equivalent to Helmholtz's equation:

$$
\frac{\partial^{2}\left(i_{Z} H\right)}{\partial x^{2}}+\frac{\partial^{2}\left(i_{Z} H\right)}{\partial y^{2}}+h^{2}\left(i_{Z} H\right)=0
$$

where $h^{2}=-\gamma^{2}+\omega^{2} \varepsilon_{r} \mu_{r}$ holds. When $\kappa^{*} i_{Z} H$ is solved for, the other geometric components $\kappa^{*} E_{\zeta}$ and $\kappa^{*} H_{\zeta}$ can be solved from Equations 35-40 [14].

\subsection{Helicoidal geometries}

Consider a magnetostatic BVP that depicts a magnetic field due to helicoidally twisted current wires (Figure 6) as a 2-D problem.

If the 3-D domain $M$ is covered with a Cartesian $x y z$-chart $f$ that replicates the observed geometry of the situation (the chart is so-called standard parameterization [6]), the orbits are helices under the chart $f$. The symmetry group $G$ is $(\mathbb{R},+)$, and the boundary values, source $J$, constitutive equation in terms of the operator $\mu$, and the cohomology conditions for $H$ and $B$ are all $G$-invariant. Therefore, the solution fields $H$ and $B$ are also $G$-invariant under the helicoidal action on $M$.

To formulate a 2-D BVP, we need a G-observer. Furthermore, for numerical solution, we need to formulate the problem on a chart. These two objectives are conveniently satisfied with helicoidal $u v w$-coordinates that are compatible with the orbits and some symmetry cell: Let $U, V$, and $W$ denote the $G$-invariant coordinate-induced basis vectors of $u_{-}, v_{-}$, and $w$-coordinates. Assume that $W$ is everywhere tangent to the orbits. Then, the coordinate differential $d w$ is the dual 1-form of $W$ such that $d w(W)=$ 


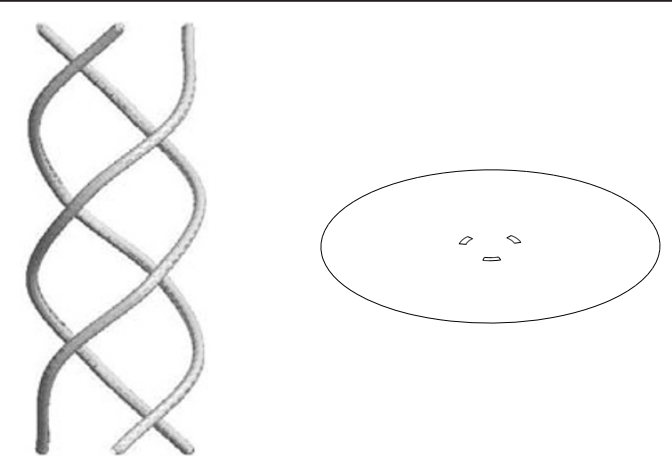

Figure 6 Helicoidal wires. Also shown is a symmetry cell, which is a plane orthogonal to the axis of rotation.

1 and $d w(U)=d w(V)=0$ hold. Thus, (W, $d w)$ is a G-observer compatible with the helicoidal coordinates such that $d w$ is defined by the $u v$-plane which is the symmetry cell. The helicoidal $u v w$-coordinates of chart $g$ are given in terms of $x y z$-coordinates of the chart $f$, when the wires are twisted around the $z$-axis, as follows:

$$
\left\{\begin{array}{l}
u=x \cos (\alpha z)-y \sin (\alpha z) \\
v=x \sin (\alpha z)+y \cos (\alpha z) \\
w=z
\end{array}\right.
$$

where $\alpha$ is the twist pitch describing the extent of twisting. Notice that the symmetry cell coincides with the $x y$-plane in the codomain of $f$.

The problem to be solved on the orbit space $N$, which can be naturally identified with the $u v$-plane, is

$$
\begin{array}{rlrl}
d_{N} H_{d w} & =J_{d w}, & i_{W} B=\mu_{d w}^{W}\left(H_{d w}\right)+\mu_{W}^{W}\left(i_{W} H\right), \\
d_{N}\left(i_{W} B\right) & =0, & & B_{d w}=\mu_{d w}^{d w}\left(H_{d w}\right)+\mu_{W}^{d w}\left(i_{W} H\right), \\
d_{N}\left(i_{W} H\right) & =0 & & \\
d_{N} B_{d w} & =0 . & &
\end{array}
$$

Here, $i_{W} J$ is zero because current density is tangent to the orbits. Furthermore, the equation $d_{N} B_{d w}=0$ is trivial. On the other hand, the equation $d_{N}\left(i_{W} H\right)=0$, together with the zero boundary condition for $i_{W} H$, implies that $i_{W} H=0$ holds. To express $\mu$ in chart $g$, we use the chart $f$, where the matrix of $\mu$ (in the coordinate-induced bases) is simply $\mu_{0} I$ (here $\mu_{0}$ is the permeability of empty space, and $I$ is the identity matrix): The matrix of $\mu$ in the chart $g$ is

$$
\mu_{g}=\frac{1}{\operatorname{det}\left(J_{c}\right)} J_{c} \mu_{f} J_{c}^{T}=\mu_{0}\left[\begin{array}{ccc}
1+\alpha^{2} v^{2} & -\alpha^{2} u v & -\alpha v \\
-\alpha^{2} u v & 1+\alpha^{2} u^{2} & \alpha u \\
-\alpha v & \alpha u & 1
\end{array}\right]
$$

where $J_{c}$ is the Jacobian matrix of the change of coordinates in (41), when $z=w=0$, in terms of the $u v w$-coordinates [[6], p. 85]. The matrices of the operators $\mu_{d w^{\prime}}^{W}$ $\mu_{d w}^{d w}, \mu_{d w}^{d w}$, and $\mu_{W}^{d w}$ in the standard basis of the $u v$-plane are (almost) the blocks of the 
matrix of $\mu_{g}$ in (42) (see Example 2). Because $i_{W} H=0$ holds, the constitutive equations are simplified, and we have the following 2-D problem to solve:

$$
\begin{aligned}
d_{N} H_{d w} & =J_{d w}, & i_{W} B & =\mu_{d w}^{W}\left(H_{d w}\right), \\
d_{N}\left(i_{W} B\right) & =0, & B_{d w} & =\mu_{d w}^{d w}\left(H_{d w}\right) .
\end{aligned}
$$

Note that the last equation is for evaluation only. The 3-D solution is then $H=H_{d w}$ and $B=B_{d w}+d w \wedge i_{W} B$ at the points of the $u v$-plane. The solution can be expanded to the whole $M$ with the pullbacks of the symmetry transformations. Calculated results can be found in [6].

\section{Conclusion}

Symmetries of BVPs make it possible to reduce the problems and thus make them easier and faster to solve. However, symmetry is often applied in engineering intuitively case-by-case basis without systematic formulations. Particularly, this is the case for dimensional reduction, which is actually based on symmetry and appears in a number of different guises.

Starting with a rigorous Definition of symmetry, we have presented a formal and systematic approach to symmetry and dimensional reduction of BVPs expressed with the exterior derivative. Particular objective has been the use of mathematical structures that are natural for each concept related to symmetries of BVPs, giving them a clear geometrical meaning. With this objective in mind, we have used tools of differential geometry, which are suitable for all dimensions, and constructed a coordinate-free theory of dimensional reduction. The theory includes sufficient conditions for dimensional reduction and a systematic procedure to formulate lower-dimensional BVPs.

The systematic approach underlies many traditional solutions. For example, timeharmonic fields, modes of waveguides, and fields due to helicoidal currents in electromagnetics can be explained in terms of the developed theory of dimensional reduction. Particularly, the systematic approach can help to recognize and apply symmetries that are not intuitively clear at the first sight and therefore can broaden the scope of applications of symmetry.

\section{Abbreviations}

BVP: boundary value problem.

\section{Author details}

'Department of Mathematics, Tampere University of Technology, P.O. Box 553, Tampere Fl-33101, Finland

2Electromagnetics, Tampere University of Technology, P.O. Box 692, Tampere FI-33101, Finland

\section{Authors' contributions}

The manuscript was drafted by PR and it is based on his PhD thesis. SS and LK were the supervisors of the thesis and gave detailed comments on the manuscript. All authors read and approved the final manuscript.

\section{Competing interests}

The authors declare that they have no competing interests.

Received: 1 March 2011 Accepted: 29 July 2011 Published: 29 July 2011

References

1. Bossavit, A: Boundary value problems with symmetry, and their approximation by finite elements. SIAM J Appl Math. 53, 1352-1380 (1993). doi:10.1137/0153064

Boothby, WM: An Introduction to Differentiable Manifolds and Riemannian Geometry. Academic Press, New York (1975)

Burke, WL: Applied Differential Geometry. Cambridge University Press, New York (1985)

Jänich, K: Vector Analysis. Springer, Berlin (2001)

Warner, FW: Foundations of Differentiable Manifolds and Lie Groups. Springer, Berlin (1983) 
6. Raumonen, P: Mathematical structures for dimensional reduction and equivalence classification of electromagnetic boundary value problems. PhD thesis, Tampere University of Technology. http://URN.fi/URN:NBN:fi:tty-200908196872 (2009)

7. Gross, PW, Kotiuga, PR: Electromagnetic Theory and Computation: A Topological Approach. Cambridge University Press, Cambridge (2004)

8. Hehl, F, Obukhov, Y: Foundations of Classical Electrodynamics: Charge, Flux, and Metric. Brickhäuser, Basel (2003)

9. Olver, PJ: Applications of Lie Groups to Differential Equations. Springer, Berlin (1986)

10. Geroch, R: Mathematical Physics. University of Chicago Press, Chicago (1985)

11. Fecko, M: On 3+1 decompositions with respect to an observer field via differential forms. J Math Phys. 38, 4542-4560 (1997). doi:10.1063/1.532142

12. Kurz, S, Auchmann, B, Flemisch, B: Dimensional reduction of field problems in a differential-forms framework. COMPEL. 28, 907-922 (2009)

13. Montgomery, D: What is a topological group? The American Mathematical Monthly. 52, 302-307 (1945). doi:10.2307/ 2305290

14. Cheng, DK: Fundamentals of Engineering Electromagnetics. Prentice-Hall, Upper Saddle River (1993)

doi:10.1186/1687-2770-2011-9

Cite this article as: Raumonen et al:: Dimensional reduction of electromagnetic boundary value problems.

Boundary Value Problems 2011 2011:9.

\section{Submit your manuscript to a SpringerOpen ${ }^{\odot}$} journal and benefit from:

- Convenient online submission

- Rigorous peer review

- Immediate publication on acceptance

- Open access: articles freely available online

- High visibility within the field

- Retaining the copyright to your article

Submit your next manuscript at $\boldsymbol{s p r i n g e r o p e n . c o m ~}$ 